\title{
Design of Energy Management System using Autonomous Hybrid Micro-Grid under IOT Environment
}

\author{
S.Sivakumar, K.Siddappa Naidu, K.Karunanithi
}

\begin{abstract}
In this paper, the automated power consumption unit is implemented by smart system technology. The interfacing could be done between the user and power sensing unit in which the collection of data is segregated and processed by the common interface bridge unit. Then, the data is transmitted to the power grid unit where the the processing and sensing power consumption is executed for the consumer in aspects of bill generation. All this kind of environment is controlled by the Internet of Things (IoT) substances and regulated by the configured power supplies. The entire setup wire diagram is simulated using Lab VIEW. The another advantage of the proposed system is equipped with highly cyber security point.
\end{abstract}

Keyword: conditions, cyber security, LabVIEW IOT(gmail), GSM, data monitoring

\section{INTRODUCTION}

In this upcoming technology IoT is the innovative technology which is the net device that can be adapted to the human user appliances that can be actuators, electronics and software where the data are exchanged. This technology will bring the convenience to access and monitor the devices anywhere. This technology involves internet connectivity apart from the standard devices such as smart phone, laptop and desktops at any range.

The IoT working environment consists of web accessible inbuilt smart things, communication hardware and sensors. These smart devise will collect data and send to their working environment. The major process of the IoT devices is to collect the various changes that occurred in the implemented device guidance of sensors and pass through the gateway of the IoT to send change of data to the server. This device will not process without human intervention even though client can interact with their device. The protocol used in the IoT to enable the device is networking, communication and connectivity protocols.

Revised Manuscript Received on June 22, 2019.

S.Sivakumar, Research Scholar, Department of EEE, Vel Tech Rangarajan Dr.Sagunthala R\&D Institute of Science and Technology, Avadi, Chennai, Tamilnadu-600062, India.

K.SiddappaNaidu, Professor Emeritus, Department of ECE, Vel Tech RangarajanDr.Sagunthala R\&D Institute of Science and Technology, Avadi, Chennai, Tamilnadu-600062, India.

K.Karunanithi, Associate Professor, Department of EEE, Vel Tech Rangarajan Dr.Sagunthala R\&D Institute of Science and Technology, Avadi, Chennai, Tamilnadu-600062, India.

\section{Wind Power}

Wind energy is the renewable energy that is generated by the air runduring wind turbines that supply the mechanical potential power to change electric generator. It is the alternative source of the fossil fuel that will help the eco system for reducing the pollution. There will be the turbans that will help to generate the swing to spin the machine that will produce the electricity which reduce the consumption. This energy power generation is up growing alternative electric source.

\section{Solar Power}

The most peak renewable energy source is the solar power where the energy is generated by the sunlight using the photovoltacis(PV) that is indirectly concentrated solar power. This system use lenses and tracking system that focus a huge sunlit in tiny beam that convert into electric power.In the developing and developed nations are developing this energy resource to reduce the pollution and save the environment protection. It bring the innovative idea for developing the energy source.

\section{Government Power Grid}

Power Grid Corporation of India Limited (POWERGRID) built-in on 23 October 1989 in the Companies Act, 1956 as a community imperfect company, wholly owned by the Government of India. Its novel name was the 'National Power Transmission Corporation Limited', and it was exciting with executing, preparation, functioning owning, and maintaining high-voltage broadcast systems in the country. On 8 November 1990, the National Power Transmission companies inward its official document for beginning of industry. Their forename was then distorted to Power Grid Corporation of India Limited, which took effect on 23 October 1992.

\section{Cloud Computing}

The kind of sharing the resource that are configure the system resource and the high level service that is rapidly provisioned with low management effort through the internet. It relies on sharing of resource that achieves coherence and the public utility. The basic services provided by the cloud are IAAS, PAAS and SAAS. 2. 


\section{LITERATURE SURVEY}

In this paper [1] aims to establish an Arduino and IOT based Multi Agent System (MAS) for rising require side reply of a micro-grid. High diffusion of renewable energy possessions in the present grid is becoming crucial. To meet the stochastic nature of the environment and dynamic loadings, new organization and manage approaches are necessary. Here, a Multi Agent System is used for an makeshift, strengthened, dispersed, autonomous energy management of the micro-grid in arrange to animatedly and flexibly adapt to the changes in the surroundings. The micro-grid contains two units; each unit has a solar Photo Voltaic (PV) system, a wind unit, restricted customer, and a battery. For lively and effectual power administration, a simulation model is developed in Java Agent Development Environment (JADE) to take knowledgeable decisions and to decide the most possible act for stabilize and ornamental the micro-grid.

In this paper [2] proposes a smart grid (SG) has involved great notice due to new ecological problems. SG technologies allow users, such as energy system operators and clients, to decrease energy expenditure and the release of conservatory gases, by altering energy communications more professionally. As a part of the SG, home energy management system (HEMS) has turn out to be more and more significant, since energy consumption of housing division financial records for a important quantity of total energy consumption. However, a conservative HEMS has some architectural limits on scalability, reusability, and interoperability.

In this paper [3] equipment MAS in LabVIEW environment for dispersed, independent energy hybrid management of a solar-wind and micro-grid. A network linked solar micro-grid, which has solar flex sheets and battery., power consumption of solar energy module of two solar units are deliberate. Every constituents of the microgrid is in use as a manager and these agents get choice separately as well as together for optimal energy management. The planned move toward separately manage the dynamics due to irregular nature of solar power, chance of load, lively pricing of network and decide the best likely act to steady and optimize the solar micro-grid. MAS have the suppleness for stop and engage in recreation and so solar power and load is additional or detached flawlessly with no moving the micro-grid operations. In addition, MAS increases the ready competence, most important to financial and ecological optimization of solar micro-grid.

In this paper [4] proposes Energy management which is more important and complex in recent days.In this paper [5] suggest a Hybrid microgrid organization consisting of renewable energy source like storm and small hydro the length of with AC/DC load system linked by bidirectional converter is future in this paper. A hybrid system consisting of storm, hydro, storage systems and grid is model and replicated using MATLAB/SIMULINK® for dissimilar scenarios of changeable weight insist profile and basis difference. Manage algorithm and power organization of dispersed sources is future for achieve smooth move of power and steady process of system. To steady the cross system, put load, energy depot system used as feasible means. As of imitation results it is establish that future wind hydro system performs adequately under dissimilar lively circumstances to uphold electrical energy and incidence within the limitations. Simulation results validate the output of LabVIEW data which is obtained from micro-hybrid grid.

In this paper [6] Microgrids are a possible trail to organize smart grids, an clever and extremely automatic power system. Their processes stress a devoted announcement communications to direct, control and monitor everything. Therefore, elegant devices will be linked to hold up the enlargement of grid elegance raising the dependency on message networks, which consume a far above the ground amount of power. In an energy-limited scenario, one of the major issues is to improve the authority supply time. Therefore, this paper demonstrates the microgrid energy management, included with a message communications to get better optimization process at utmost energy competence.

\section{METHODOLOGY AND IMPLEMENTATION}

\section{Photovoltaic system}

In this block, the solar energy properly captured by the provided solar panels and it is procured by the DC-DC system where the net solar energy is preserved and stored in a lithium battery for the uninterrupted performances of autonomous grid system

\section{The wind turbine system}

The quite inhabitancy of this block is used to convert the rotational energy to the electrical energy. It could be obtained by the provided mini fan blades. The converted electrical energy is properly directed to the storage cells. The loading point of the storage cells is fixed as DC-AC converter. The main objective of this unit is furnish the AC supply through the installed inverted to the home devices.

\section{Time Scheduling and Power Consumption:}

Time Scheduling and Power Consumption: Load complementary or load distribution is a plan to distribute workload in the middle of processors in a dispersed system. A few literatures compare both load balancing and load sharing. Load balancing is frequently distinct as a plan which attempts to promise that every computer in a system has equivalent load. Load sharing is constructed using LabVIEW and embedded system. This stratergy have the similar goal, which is to make improved use of the scheme resources (usually the $\mathrm{CPU}$ ) by making sure that no nodes are idle.

\section{Customer model}

The customer model section is the heart unit of the proposed hybrid power grid house. Since the usage of power consumption is differed for customer to customer, the grid house need to allocated the power sources according to the end user. The variation of power consumption is dictated by the available storage cell. The whole day and power consumption by the each user is purely monitored by the

Published By: 
hybrid screening volatile system which provides the simple transfer mail protocol and it tends to help for finding user consumption rate community. This blocks also acts a home gateway protocol and assigns the pocket of data to be assigned when the customer needs excess power.

\section{Cost Function}

In this section, the bill cost for the power consumption is calculated. The bill monitoring process is divided by two nodes. One is fog node $(\mathrm{F})$ and slot node $(\mathrm{S})$. The f node is referred for the user interface to the central management unit and $\mathrm{S}$ node is used for the making time allocation and priority for the user consuming duration. As a whole, both the nodes are involved to setup the effective utilization cost value enumeration without excess bill generation for the right user at actual power usage.

\section{Prediction}

The data prediction with respect to the user availability, range of utility, resources available and pocket size with the ground node. In this system, the accurate linear time bound series model with linear variant, root mean square method has been followed. The prediction factor is derived from the past and present mean error value and the prediction pattern is applied between the power source to the PV system.Few other prediction pattern also available such that auto regression and auto correlation analysis, cross regression and cross correlation analysis.

\section{Keil Compiler}

The Keil micro C51 for ARM is the well-liked $8051 \mathrm{C}$ compiler. ... The C51 Compiler translates $\mathrm{C}$ foundation records into reloadable thing modules which hold full representative in order for debugging with the $\mu$ Vision Debugger or an in-circuit emulator. The Keil 8051 Development Tools are intended to resolve the multifaceted problems opposite entrenched software developers.

While initial a new project, just choose the microcontroller as more efficient

\section{Mplab X-Ide Compiler}

MPLAB is included expansion surroundings for the growth of entrenched applications on PIC and dsPIC microcontrollers, and is urbanized by Microchip skill. MPLAB $X$ is the latest description of MPLAB, and is urbanized on the NetBeans stage. MPLAB and MPLAB X hold up scheme organization, cipher restriction, debug and indoctrination of Peripheral interface controller and ARM microcontrollers,.

\section{RESULTS AND DISCUSSION}

The simulated results with experimental demonstration are discussed detail in this section. In IoT block, BME280 temperature humidity/pressure sensor, MQ-2 gas sensor, light sensor, ACS 712 current sensor, 5-V relay, and an ESP 8266 Huzzah MCU/WiFi controller has been implemented.An HTTP/CoAP is fixed with gate way protocol and it is configured using the python3.6.2. the communication establishment between the sensors and user data is done by HTTP. The implementation test bed and the communication protocol stack are shown in Fig. 4.1.Fig. 4 shows the detail algorithm of the home gateway in the HTTP operation mode. As it is shown in this figure, at a regular time interval (15 s), the gateway makes an HTTP connection to the IoT node and send the GET method. The GET method contains the URI of the requested resources. After that, the gateway gets the attention from the sensor node. When the response pin reaches $2000 \mathrm{~K}$, the payload will be activated and gives its response to the sensor node where the raw data is collected in order to satisfy the payload response. Later, the gateway gives the connection establishment between the HTTP to FOG newtwork using JAVA script. The fog network assigns the token for the priority slot and moves to the cloud node with the frequency $15 \mathrm{~s}$. Then both connections are closed. This is frequenty repeated each $15 \mathrm{~s}$, periodically. The same procedure is applied for CoAP protocol.

The only difference is that as CoAP uses the UDP protocol as transport layer protocol which is unreliable, there is no guaranty for a successful connection. The consecutive response are properly screened and process by the sensor node, fog node and cloud node. The connection establishment was successfully launched using SMTP and HTTP protocols. / FIG: 4.1 CYBER SECURITIES / FIG: 4.2 Data Segregation Unit / FIG 4.3 IOT 1 / FIG 4.4 IOT 2 / FIG 4.5 IOT 3 / FIG 4.6 Receiver side / Fig 4.7 Source triggering / Fig 4.8 Time scheduling unit / Fig 4.9 Time scheduling unit / Fig 4.10 Check new min delay.ver1.vi block diagram / Fig 4.11 Check New min delay.VER1.vi Front Panel / Fig 4.12 Check New min delay.VER1.vi Front Panel_2 / Fig 4.13 Check New min delay.VER1.vi Front Panel_3 / Fig 4.14 Check New min delay.VER1.vi Front Panel_4 / Fig 4.15 Check New min delay.VER1.vi Front Panel_5 / Fig 4.16 Check New min delay.VER1.vi Front Panel_6 5.

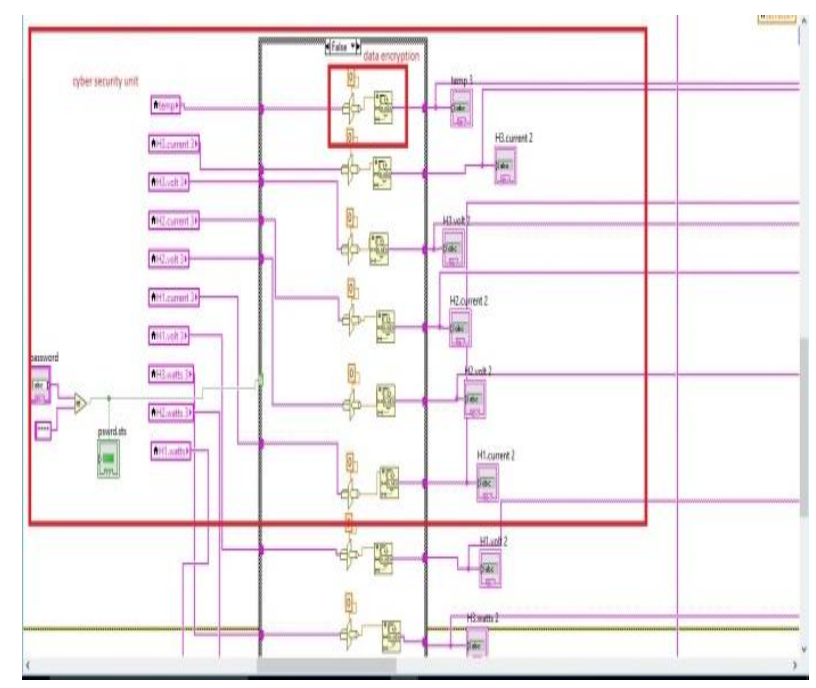

Fig. 4.1 Cyber Securities 
Design of Energy Management System using Autonomous Hybrid Micro-Grid under IOT Environment

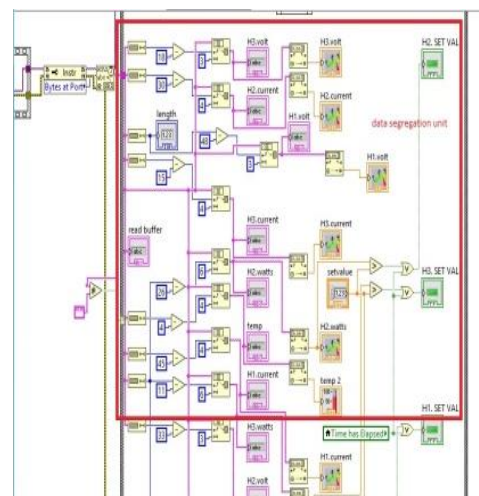

Fig. 4.2 Data Segregation Unit

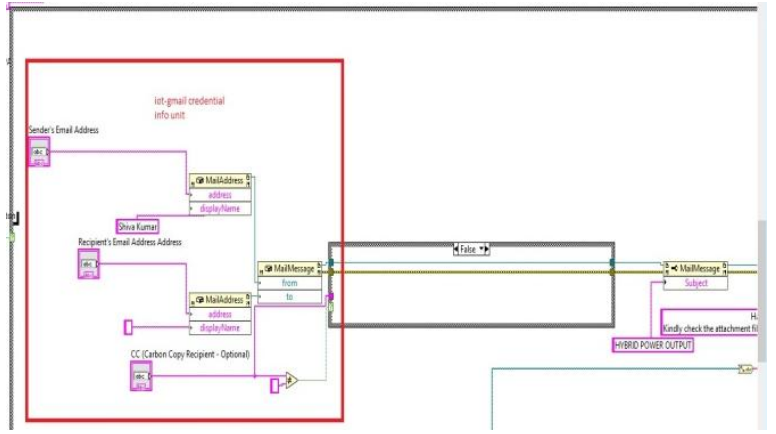

Fig. 4.3 IOT 1

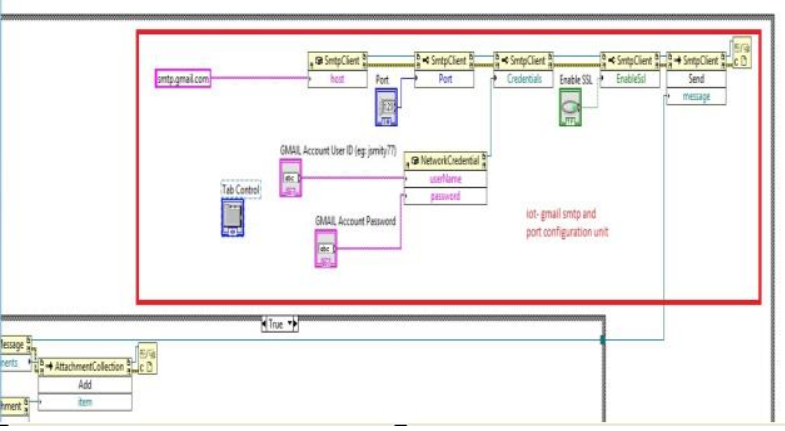

Fig. 4.4 IOT 2

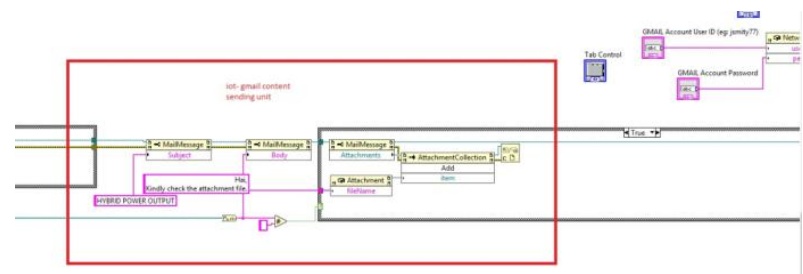

畐,

Fig. 4.5 IOT 3

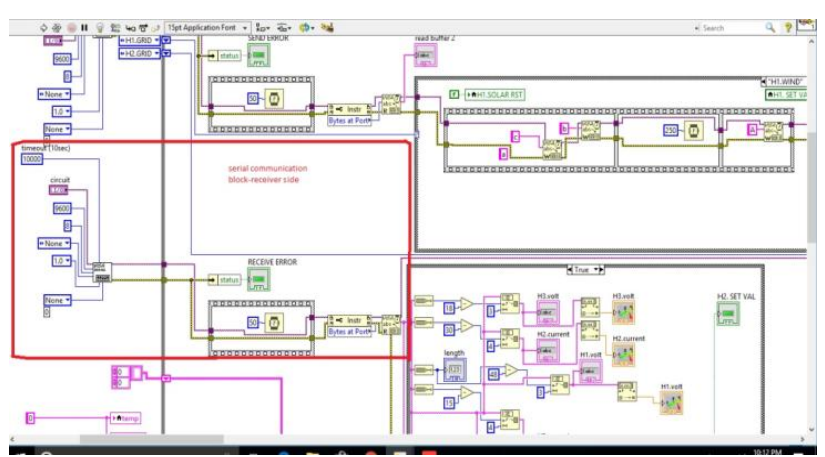

Fig. 4.6 receiver side

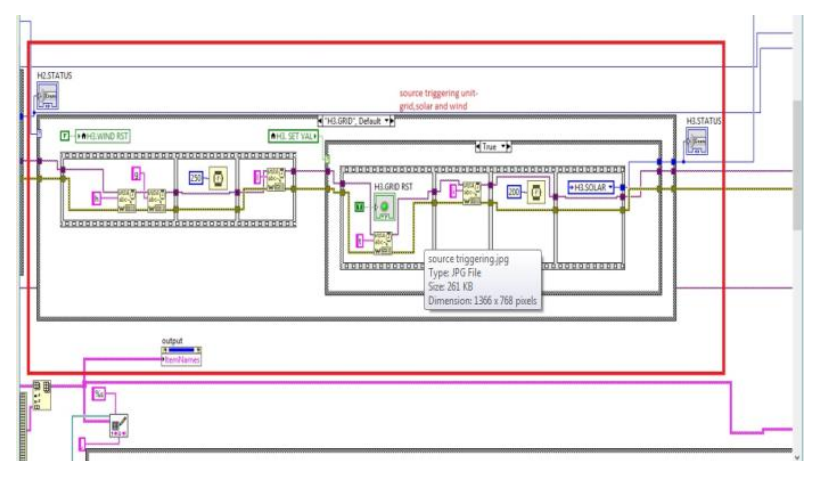

Fig. 4.7 source triggering

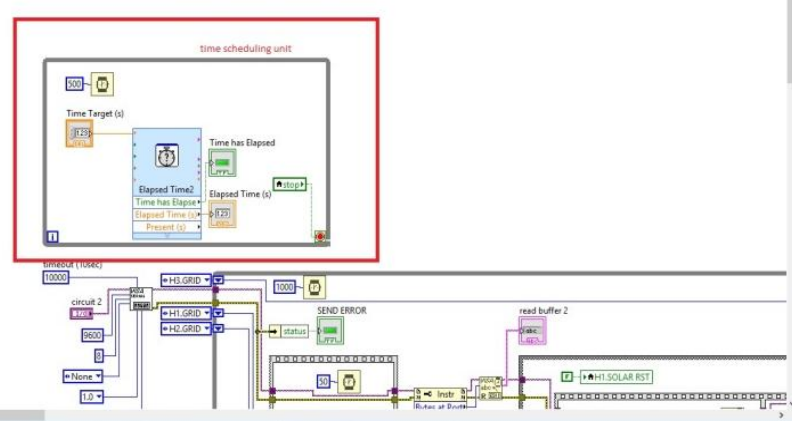

Fig. 4.8 time scheduling unit

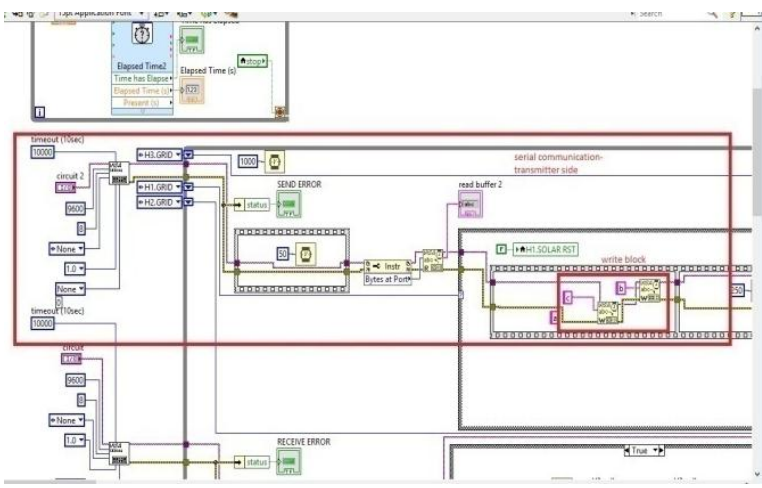

Fig. 4.9 time scheduling unit

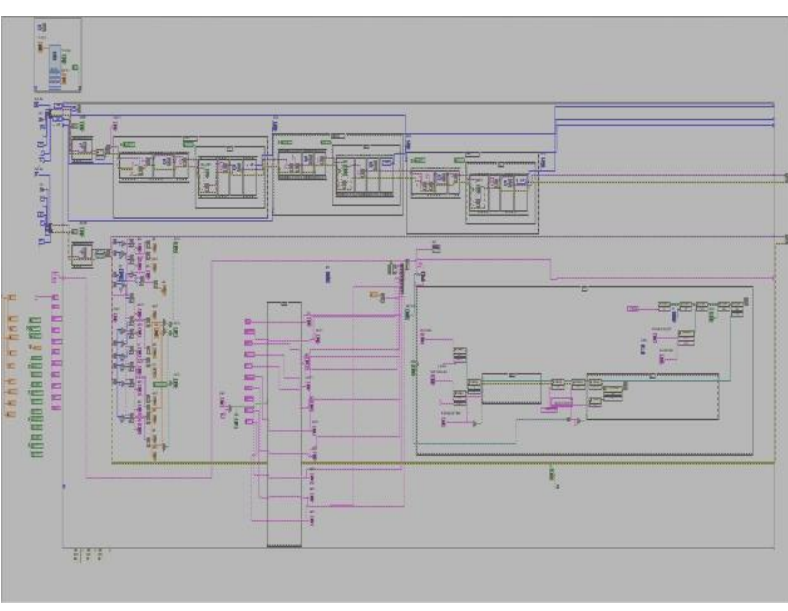

Fig. 4.10 check new min delay.ver1.vi block diagram

Blue Eyes Intelligence Engineering

\& Sciences Publication

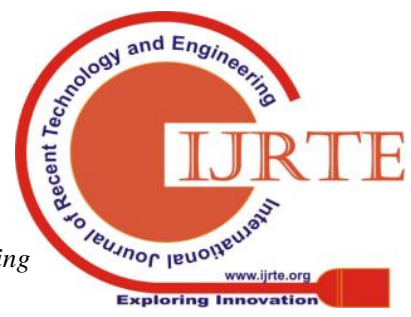




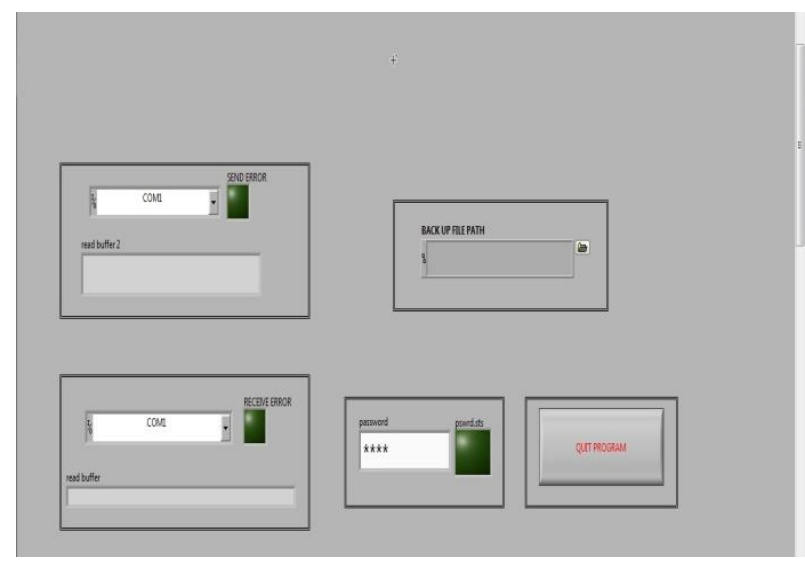

Fig. 4.11 check new min delay.ver1.vi front panel

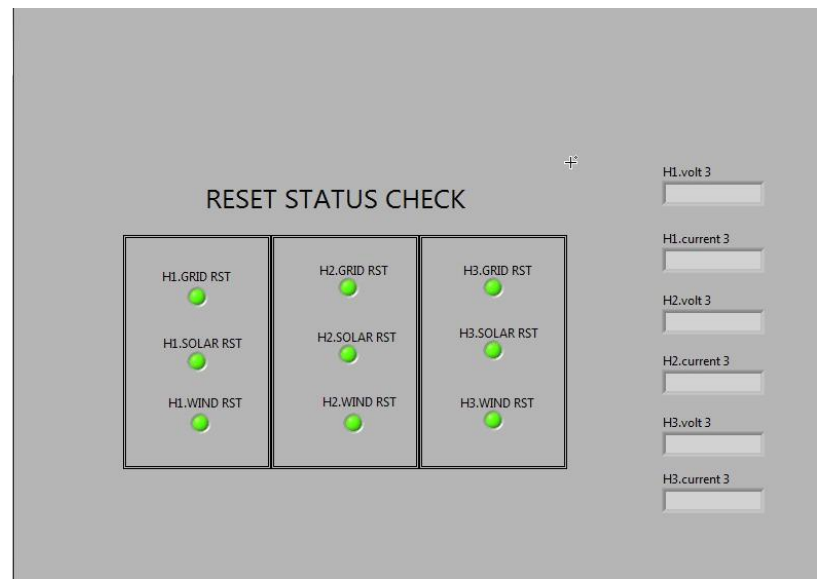

Fig. 4.12 check new min delay.ver1.vi front panel_2

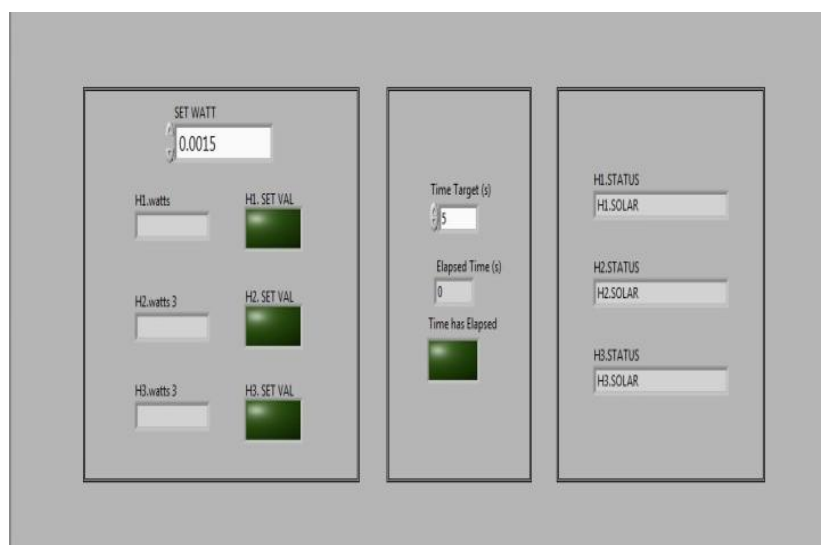

Fig. 4.13 check new min delay.ver1.vi front panel_3

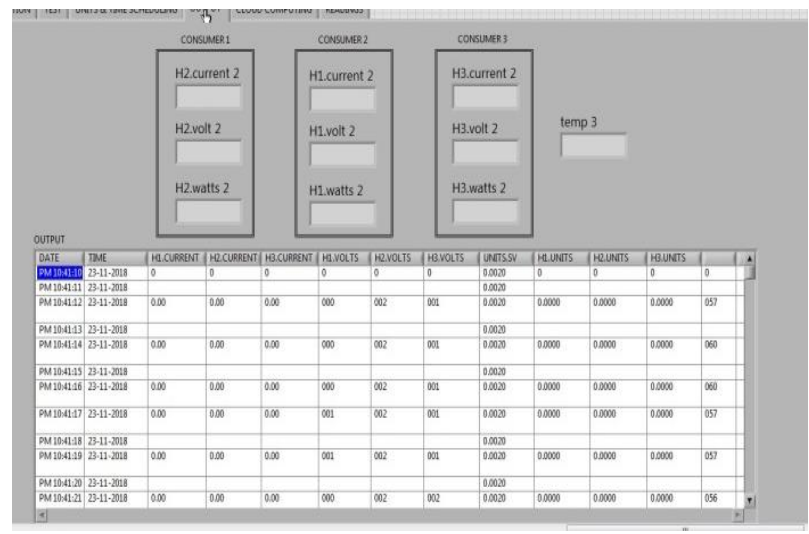

Fig. 4.14 check new min delay.ver1.vi front panel_4

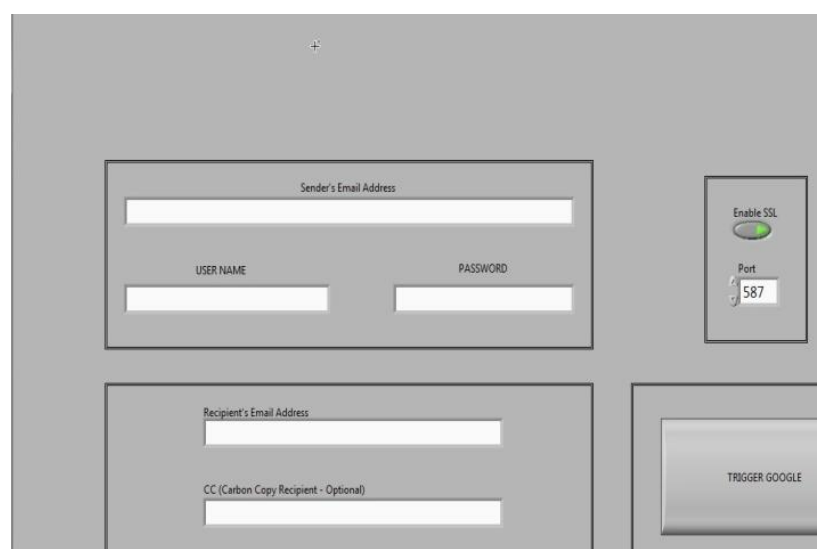

Fig. 4.15 check new min delay.ver1.vi front panel_5

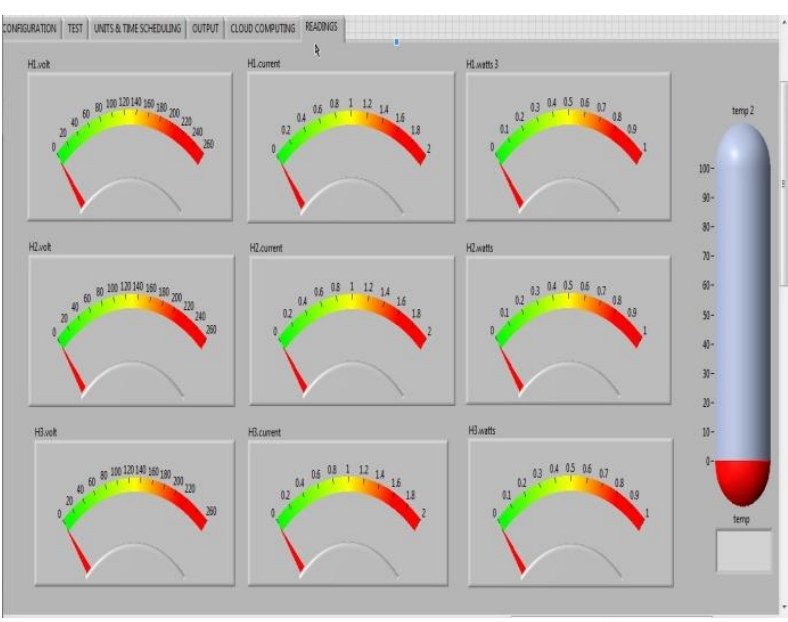

Fig 4.16 check new min delay.ver1.vi front panel_6

\section{CONCLUSION}

The automated power consumption unit was implemented by smart system technology. The interfacing was done between the user and power sensing unit in which the collection of data was segregated and processed by the common interface bridge unit. Then, the data is then transmitted to the power grid unit where the processing and sensing power consumption is executed for the consumer in aspects of bill generation. All this kind of environment was controlled by the Internet of Things (IoT) substances and regulated by the configured power supplies. The entire setup wire diagram is simulated using Lab VIEW. The another advantage of the proposed system was equipped with highly cyber security point.

\section{REFERENCES}

1. Y. Lee et al., "A modular $1 \mathrm{~mm} 3$ die-stacked sensing platform with lowpower I2C inter-die communication and multi-modal energy harvesting,"IEEE J. Solid-State Circuits, vol. 48, no. 1, pp. 229-243, Jan. 2013.

2. Y. Zhang et al., "A batteryless $19 \mu \mathrm{W}$ MICS/ISM-band energy harvesting body sensor node SoC for ExG applications," IEEE J. SolidStateCircuits, vol. 48, no. 1, pp. 199-213, Jan. 2013.

3. S. Bandyopadhyay and A. P. Chandrakasan, "Platform architecture forsolar, thermal, and vibration energy combining with MPPT and singleinductor," IEEE J. Solid-State Circuits, vol. 47, no. 9, pp. 21992215,Sep. 2012.

\section{Published By:}

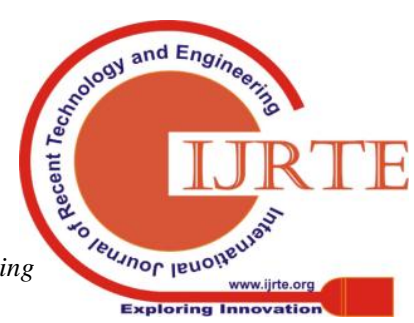


4. S. Bandyopadhyay, P. P. Mercier, A. C. Lysaght, K. M. Stankovic, andA. P. Chandrakasan, "A $1.1 \mathrm{nW}$ energy-harvesting system with 544 pWquiescent power for next-generation implants," IEEE J. SolidStateCircuits, vol. 49, no. 12, pp. 2812-2824, Dec. 2014.

5. Y. K. Teh and P. K. T. Mok, "Design of transformer-based boost converterfor high internal resistance energy harvesting sources with 21 $\mathrm{mV}$ self-startup voltage and 74\% power efficiency," IEEE J. SolidState Circuits, vol. 49, no. 11, pp. 2694-2704, Nov. 2014.

6. W. Jung et al., "An ultra-low power fully integrated energy harvesterbased on self-oscillating switched-capacitor voltage doubler," IEEE J.Solid-State Circuits, vol. 49, no. 12, pp. 2800-2811, Dec. 2014.

7. Bosch BMP180 Digital Pressure Sensor,, 2015., http://aebst.resource.bosch.com/media/products/dokumente/bmp180/BSTBMP180-DS000-12 1.pdf.

8. CAT34TS00 1.8 V Digital Temperature Sensor, On Semiconductor 2014,[9] Data Sheet, SHTC1 Humidity and Temperature Sensor IC, ver.4,SensirionAug.2015,http://www.sensirion.com/fileadmin/user_upl oad/customers/sensirion/Dokumente/Humidity/Sensirion_Humidity_S HTC1_Datasheet_V3.pdf.

9. "What the Internet of Things (IoT) Needs to Become Reality," FreescaleSemiconductor/ARM, Inc. White Paper, 2012-2014

10. R. Yu and T. Watteyne, "Reliable, low power wireless sensor networksfor the internet of things: Making wireless sensors as accessible as webservers," http://cds.linear.com/docs/en/whitepaper/wp003.pdf.

11. X. Liu and E. Sanchez-Sinencio, “A 0.45-to-3V reconfigurable chargepump energy harvester with two-dimensional MPPT for Internet ofThings," IEEE Int. Solid-State Circuits Conf. Dig. Tech. Papers, 2015,pp. 1-3.

12. G. Palumbo and D. Pappalardo, "Charge pump circuits: An overview ondesign strategies and topologies," IEEECircuits Syst. Mag.,vol. 10,no.1,pp. 31-45, 2010.

13. E. J. Carlson, K. Strunz, and B. P. Otis, "A $20 \mathrm{mV}$ input boost converterwith efficient digital control for thermoelectric energy harvesting," IEEEJ. Solid-State Circuits, vol. 45, no. 4, pp. 741-750, Apr. 2010

14. P. S. Weng, H. Y. Tang, P. C. Ku, and L. H. Lu, "50 mV-input batteryless boost converter for thermal energy harvesting," IEEE J. SolidStateCircuits, vol. 48, no. 4, pp. 1031-1041, Apr. 2013.

15. Y.-C. Shih and B. P. Otis, "An inductorless DC-DC converter for energyharvesting with a $1.2 \mu \mathrm{W}$ bandgap-referenced output controller," IEEETrans. Circuits Syst. II, Exp. Briefs, vol. 58, no. 12, pp. 832-836, Dec.2011.

16. J.Zarate-Roldan,S.Carreon-Bautista,A.Costilla-Reyes,andE.SanchezSinencio, "An ultra-low power power management unit with -40 dBswitching-noise-suppression for a $3 \times 3$ thermoelectric generator arraywith 57\% maximum end-to-end efficiency," Proc. IEEE Custom Integr.Circuits Conf., San Jose, CA, USA, 2014, pp. 1-4.

17. I. Doms, P. Merken, C. Van Hoof, and R. P. Mertens, "Capacitivepower management circuit for micropower thermoelectric generatorswith a $1.4 \mu \mathrm{A}$ controller," IEEE J. Solid-State Circuits, vol. 44, no. 10,pp. 2824-2833, Oct. 2009.

18. Y. H. Lee et al., "A low quiescent current asynchronous digital-LDO withPLL-modulated fast-DVS power management in $40 \mathrm{~nm}$ SoC for MIPSperformance improvement," IEEE J. Solid-State Circuits, vol. 48, no. 4,pp. 1018-1030, Apr. 2013.

19. S. Gangopadhyay, D. Somasekhar, J. W. Tschanz, and A. Raychowdhury,"A32nmembedded,fulldigital,phaselockedlowdropoutre gulatorforfine grained power management in digital circuits," IEEE J. Solid-StateCircuits, vol. 49, no. 11, pp. 2684-2693, Nov. 2014. 